\title{
Diversidade de moscas frugívoras (Diptera, Tephritoidea) em áreas de matas decídua e ciliar no Pantanal sul-mato-grossense, Brasil
}

\author{
Edima Ramos Minzão ${ }^{1} \&$ Manoel Araécio Uchôa-Fernandes ${ }^{1}$
}

\begin{abstract}
${ }^{1}$ Laboratório de Insetos Frugívoros, Programa de Pós-graduação em Entomologia e Conservação da Biodiversidade, Faculdade de Ciências Biológicas e Ambientais, Caixa Postal 241, Universidade Federal da Grande Dourados (UFGD), 79804-970 Dourados-MS.

uchoa.fernandes@ufgd.edu.br; e_minzao@yahoo.com.br
\end{abstract}

\begin{abstract}
Diversity of frugivorous flies (Diptera, Tephritoidea) in areas of decidual and riparian forests in South Pantanal, Brazil. The knowledge of fly species diversity and population patterns in the ecosystems is important to subsidy the choice of ecologically correct methods for control of tephritoid pests. The aim of this paper is to evaluate the diversity of tephritoids and their population patterns in a decidual and a riparian forest. Flies were caught in McPhail traps with food bait in two natural forest reserves at the Municipality of Corumbá-MS, from August 2003 to August 2004. Thirteen species belonging to five genera and two families were recorded. At Sítio Pingo de Amor (decidual forest [DF]), were collected: Anastrepha dissimilis, A. fraterculus, A. obliqua, A. rheediae, A. sororcula, A. undosa and Ceratitis capitata (Tephritidae), besides Dasiops sp.1, Dasiops sp.2, Lonchaea sp.1, Lonchaea sp.2, Neosilba sp.1 and Neosilba sp.2., from Lonchaeidae. At Canal do Tamengo (riparian forest [RF]), were recorded all species above mentioned, except: A. dissimilis, A. rheediae, A. undosa, Dasiops sp.2 and Neosilba sp.2. The Shannon-Weaver diversity index (H'), was 2.01 in the DF and 1.51 in the RF. Anastrepha obliqua was very abundant in both natural forest reserves. In the DF A. sororcula was constant and predominant and, Neosilba sp.1 was very abundant. At both environments A. obliqua, Lonchaea sp.2 and Neosilba sp.1 were very frequent and, A. obliqua and Neosilba sp.1 were dominant.
\end{abstract}

KEYWORDS. Faunistic studies; Lonchaeidae; McPhail traps; Tephritidae.

RESUMO. Diversidade de moscas frugívoras (Diptera, Tephritoidea) em áreas de matas decídua e ciliar no Pantanal sulmato-grossense, Brasil. O conhecimento da diversidade de espécies de moscas nos ecossistemas é importante para subsidiar na escolha de métodos ecologicamente corretos para o controle de tefritóideos (Tephritidae e Lonchaeidae) pragas. O objetivo deste trabalho foi avaliar a diversidade de tefritóideos e seus padrões populacionais em áreas de matas decídua e ciliar. As moscas foram capturadas em armadilhas McPhail com atrativo alimentar em duas reservas florestais do município de Corumbá-MS, de agosto de 2003 a agosto de 2004. Treze espécies pertencentes a cinco gêneros e duas famílias foram registradas. No Sítio Pingo de Amor (mata decídua [MD]), foram coletadas: Anastrepha dissimilis, A. fraterculus, A. obliqua, A. rheediae, A. sororcula, A. undosa e Ceratitis capitata (Tephritidae) e de Lonchaeidae foram capturadas: Dasiops sp.1, Dasiops sp.2, Lonchaea sp.1, Lonchaea sp.2, Neosilba sp.1 e Neosilba sp.2. No Canal do Tamengo (mata ciliar [MC]), foram obtidas todas as espécies mencionadas acima, exceto: A. dissimilis, A. rheediae, A. undosa, Dasiops sp.2 and Neosilba sp.2. O índice de diversidade de Shannon-Weaver (H'), foi: 2,01 na MD e 1,51 na MC. Anastrepha obliqua foi caracterizada como muito abundante em ambas as reservas florestais. Na mata decídua $A$. sororcula foi constante e predominante e, Neosilba sp.1, muito abundante. Em ambos os ambientes A. obliqua, Lonchaea sp.2 e Neosilba sp.1 foram muito freqüentes e, A. obliqua e Neosilba sp.1 foram dominantes.

PALAVRAS-CHAVE. Armadilhas McPhail; Estudos faunísticos; Lonchaeidae; Tephritidae.

As moscas frugívoras (Tephritidae e Lonchaeidae) causam sérios prejuízos ao mercado interno de frutas e hortaliças por ocasionarem a queda precoce e/ou sua desvalorização para o consumo in natura e industrialização. Os prejuízos se refletem também no mercado externo, devido às restrições quarentenárias impostas pelos países importadores (Duarte \& Malavasi 2000). Ainda há escassez de estudos básicos que possam subsidiar programas de manejo populacional destas moscas, principalmente, aqueles sobre biologia e ecologia de populações.

As infestações de diversas espécies de frutos por Tephritoidea têm ocorrido no mundo todo, apesar dos procedimentos de quarentena adotados por vários países (Duyck et al. 2004). Tephritidae constitui um dos maiores grupos de insetos fitófagos com importância econômica mundial
(Hernández-Ortiz \& Pérez-Alonso 1993, Zucchi 2000). As espécies de Anastrepha Schiner (1868) ocorrem nos trópicos e sub-trópicos americanos (Hernández-Ortiz \& Pérez-Alonso 1993). Do gênero Ceratitis MacLeay (1829) ocorre no Brasil somente a espécie exótica, C. capitata (Wiedemann 1824), atualmente cosmopolita. Zucchi (2000) destaca que esta é uma das principais pragas das frutíferas brasileiras; infesta mais de 350 espécies de frutos, incluindo café, laranja, pêra, goiaba e várias outras culturas de importância econômica.

Os Lonchaeidae infestam frutos e flores de várias espécies de plantas cultivadas e silvestres. Espécies do gênero Neosilba McAlpine (1962) (Lonchaeinae) são pragas em citros (Citrus spp.), goiaba (Psidium guajava L.), mamão (Carica papaya L.), acerola (Malphigia emarginata D. C.), pequi (Caryocar brasiliense Camb.), entre outras. Já as espécies de Dasiops 
Rondoni (1856) (Dasiopinae), infestam frutos e flores de várias espécies de maracujás (Passiflora spp.) (Uchôa-Fernandes et al.2002).

As análises faunísticas de moscas frugívoras são escassas na literatura e limitam-se, principalmente, aos trabalhos realizados no Brasil (Canal et al. 1988; Garcia et al. 2003; UchôaFernandes et al. 2003b; Canesin \& Uchôa-Fernandes 2007). Todavia, têm ocorrido avanços no conhecimento da diversidade das moscas frugívoras por meio de estudos nos diferentes estados brasileiros, com base em inventários de espécies capturadas em armadilhas McPhail, associação com frutos hospedeiros e inimigos naturais (Zucchi 2000), bem como, em outros países sul-americanos: Nicarágua (NiklausRuiz \& Basedow 1997), México (Aluja et al. 2003), Argentina (Ovruski et al. 2005), entre outros.

O conhecimento da diversidade de espécies de moscas frugívoras em uma região é de fundamental importância para subsidiar as táticas de controle desses grupos de insetos (Araújo et al. 2005). As informações sobre essas moscas devem avançar com estudos sobre espécies não-pragas, comparações entre espécies primitivas e derivadas e sobre as populações em áreas nativas ou adjacentes aos pomares comerciais (Canesin \& Uchôa-Fernandes 2007). Nestes locais, as populações aumentam antes de invadirem os pomares. Tanto no Brasil quanto em outros países, são poucos os trabalhos conduzidos em ambientes naturais ou semi-naturais (Hernández-Ortiz \& Pérez-Alonso 1993; Canesin \& UchôaFernandes 2007; Bomfim et al. 2007). Sob essa perspectiva desenvolveu-se este trabalho, com o objetivo de registrar a ocorrência das espécies de moscas frugívoras no Pantanal sul (Corumbá), realizar o estudo faunístico e determinar a flutuação populacional dessas moscas em duas reservas florestais naturais daquele município.

\section{MATERIALE MÉTODOS}

Esta pesquisa foi desenvolvida em duas reservas florestais

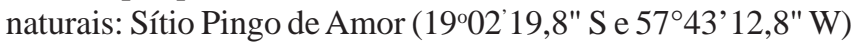
(mata decídua), a $121 \mathrm{~m}$ altitude, e em um trecho de mata ciliar da margem direita do Rio Paraguai, denominado Canal do Tamengo (1900'51,4" S e 57²1'22,9" W), a 98m altitude. Estas distam cerca de 5,0 e $1,5 \mathrm{~km}$ do perímetro urbano de CorumbáMS, respectivamente. A mata decídua compreende uma área de aproximadamente cinco hectares de flora nativa, com característica de floresta estacional decídua, e o Canal do Tamengo (6ha), consiste de vegetação ciliar (Pott \& Pott 1994).

A região apresenta temperatura média anual de $24^{\circ} \mathrm{C}$, com uma estação quente e outra fria. Os meses mais frios do ano são junho e julho, e os mais quentes setembro, outubro e novembro, este último marcando o fim da estação seca. A precipitação anual oscila de 1.000 a $1.500 \mathrm{~mm}$, concentrandose nos meses de verão, principalmente janeiro e fevereiro (Ravazzani et al. 1991).

Para a amostragem das moscas foram utilizadas oito armadilhas McPhail iscadas com hidrolisado enzimático de milho diluído a 5\% e estabilizado com bórax (Uchôa-Fernandes et al. 2003b). As armadilhas foram instaladas a 1,70m do nível do solo, sendo empregadas quatro armadilhas em cada reserva florestal. As coletas representam a soma das moscas frugívoras capturadas nas quatro armadilhas, em cada localidade, durante sete dias, a cada intervalo de 15 dias. As armadilhas eram mantidas no campo por uma semana e retiradas na semana seguinte, de agosto de 2003 a agosto de 2004. Durante cada vistoria eram lavadas e o atrativo renovado, sempre com uma semana de intervalo entre uma amostragem e a seguinte. O atrativo descartado e a água utilizada para a lavagem das armadilhas foram colocados em recipientes plásticos e descartados na rede de esgoto do laboratório.

Os tefritóideos foram fixados em álcool $70 \%$ e transportados ao Laboratório de Insetos Frugívoros da Faculdade de Ciências Biológicas e Ambientais, Universidade Federal da Grande Dourados (UFGD), Dourados-MS, para identificação. As espécies de Anastrepha foram identificadas com base, principalmente, no exame da terminália feminina, observando-se as dimensões e a morfologia do ápice do ovipositor (acúleo) e características de coloração do corpo e das asas (Zucchi 2000).

Para identificação dos Lonchaeidae foram usadas chaves e descrições (McAlpine, 1993) e em seguida morfoespeciadas. Espécimes vouchers das moscas frugívoras foram depositadas na Coleção Entomológica do Museu da Biodiversidade (insetos, plantas hospedeiras e inimigos naturais) (UFGD).

A diversidade das moscas frugívoras foi analisada por meio do índice de Shannon-Weaver (H') em cada uma das reservas florestais. De acordo com Price (1997), esse índice é amplamente utilizado em análises de diversidade de comunidades. As curvas cumulativas de espécies foram baseadas em cálculos de Chao de primeira ordem (Chao 1), com 100 aleatorizações. Para as análises de diversidade e de riqueza esperada (Chao 1), utilizou-se o Software Estimate S 6.0 (Colwell 2001).

Realizou-se a análise faunística para definir as classes de abundância, constância e dominância das espécies, de acordo Silveira Neto et al. (1976). A média e erro padrão da média do número de indivíduos coletados por espécie foram calculados, determinando-se o intervalo de confiança (IC) a $5 \%$ e a $1 \%$ de probabilidade. Foram estabelecidas as seguintes classes: ma = muito abundante (número de indivíduos maior que o limite superior do IC a 1\%); a = abundante (número de indivíduos situado entre os limites superiores do IC a $5 \%$ e a $1 \%$ ); $\mathrm{c}=$ comum (número de indivíduos situado dentro do IC a 5\%); d = dispersa (número de indivíduos situado entre os limites inferiores do IC a 5\% e a 1\%) e r = rara (número de indivíduos menor que o limite inferior do IC a $1 \%$ ). Determinou-se o intervalo de confiança da média das freqüências (\% de indivíduos coletados) com 5\% de probabilidade, adotando-se a seguinte classificação: $\mathrm{mf}=$ muito freqüente (freqüência menor que o limite superior do IC a $5 \%$ ); $f=$ freqüente (frequiência situada dentro do IC a 5\%) e pf = pouco freqüente (freqüência menor que o limite inferior do IC a 1\%) (Silveira Neto et al. 1976). A constância foi determinada para cada espécie em cada reserva florestal. As espécies consideradas predominantes foram aquelas que alcançaram os maiores 
índices faunísticos de abundância, freqüência, constância e dominância. Na análise faunística das espécies de Anastrepha foram consideradas somente as fêmeas devido a falta de chaves disponíveis para a identificação de machos.

\section{RESULTADOS}

Foram coletadas 13 espécies de cinco gêneros das famílias Tephritidae e Lonchaeidae (Tephritoidea). Tephritidae foi representada por sete espécies: seis de Anastrepha (Trypetinae), gênero mais abundante (569 indivíduos) e Ceratitis capitata (Oedaspinae), com 48 exemplares. Uma fêmea de Anastrepha teve o acúleo danificado, por isso, não foi possível a identificação. De Lonchaeidae foram obtidas seis espécies: duas de Dasiops (Dasiopinae), com 4 exemplares; duas de Lonchaea, com 33 indivíduos e duas de Neosilba (Lonchaeinae), 46 indivíduos (Tabela I).

As espécies de Tephritidae mais abundantes na mata decídua, foram: A. obliqua (Macquart, 1835) (muito abundante), A. sororcula Zucchi 1979 e A.fraterculus (Wiedemann, 1830), foram abundantes. Os lonqueídeos, Neosilba sp.1 seguida por Lonchaea sp.2 foram as espécies mais representativas. Na mata ciliar, A. obliqua, A. sororcula, C. capitata e Neosilba sp.1, foram as espécies mais abundantes (Tabela I).

Os índices de diversidade de Shannon-Weaver (H') encontrados foram: 2,01 na mata decídua e 1,51 na mata ciliar. A análise de riqueza em espécies das moscas frugívoras na mata ciliar demonstrou que a partir da $19^{\text {a }}$ coleta, a curva cumulativa de novos registros de espécies para o local atingiu a estabilidade, indicando que o esforço de coleta foi suficiente para amostrar a diversidade. Para a mata decídua, o índice observado (Sobs) foi abaixo do esperado (Chao 1), sugerindo que o número de coletas foi insuficiente para amostrar todas as espécies de moscas frugívoras naquele ambiente (Fig. 1).

\section{DISCUSSÃO}

Embora estudos quantitativos sobre riqueza de espécies de moscas frugívoras em áreas florestadas sejam escassos na Região Neotropical, a riqueza encontrada neste estudo é comparativamente menor que aquela encontrada em outras matas tropicais (Hernández-Ortiz \& Pérez-Alonso 1993; Aluja et al. 2003; Ronchi-Teles \& Silva 2005; Bomfim et al. 2007, Canesin \& Uchôa-Fernandes 2007). No entanto, deve-se destacar que ambas as áreas localizam-se na planície inundável do Pantanal e, nas duas matas, há predominância de espécies de plantas com frutos secos ou vagens (vide Pott \& Pott, 1994) - que não são hospedeiros preferenciais das moscas frugívoras (Uchôa-Fernandes, M. A., dados não publicados).

Os estudos faunísticos, por meio dos cálculos de frequiência, abundância, constância, dominância e dos índices de diversidade regional das espécies, auxiliam no entendimento dos padrões gerais de biologia, ecologia e comportamento das comunidades (Silveira Neto et al. 1976, Uchôa-Fernandes et al. 2003a).

Neste trabalho, A. obliqua foi a espécie mais freqüente (41,26\% do total de Anastrepha) na mata decídua e na mata ciliar $(54,79 \%)$ e caracterizou-se como muito abundante em ambas as reservas florestais, seguida por $A$. sororcula na mata decídua $(23,78 \%)$ e na mata ciliar $(20,74 \%)$. Esta maior frequiência e abundância de A. obliqua podem ser explicadas pela presença de várias Anacardiaceae: Mangifera indica L.
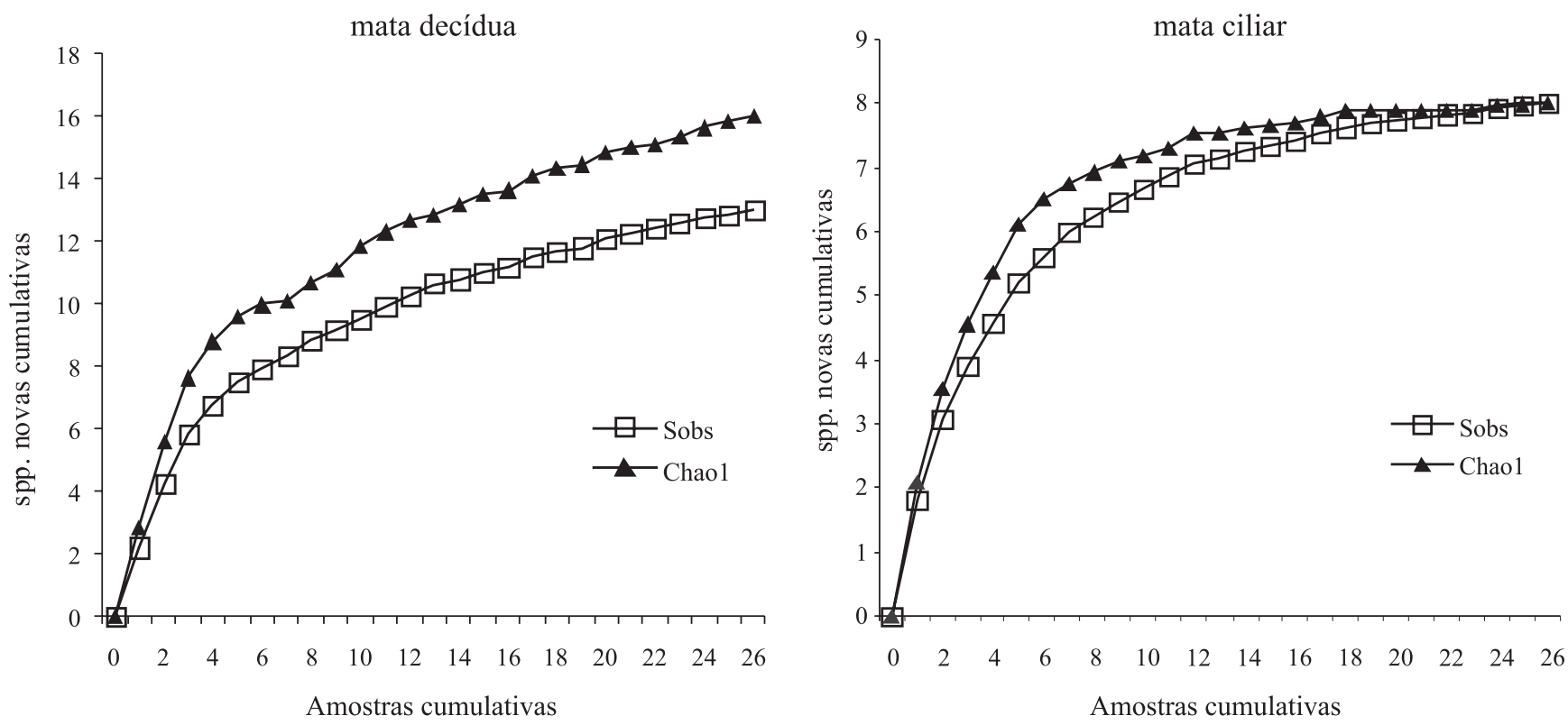

Fig. 1. Riqueza observada (Sobs) e esperada (Chao 1) para as espécies de moscas frugívoras (Diptera: Tephritoidea) capturadas com armadilhas McPhail nas reservas florestais naturais: mata decídua (Sítio Pingo de Amor) e mata ciliar (Canal do Tamengo), município de Corumbá-MS (agosto/2003 a agosto/2004). 
Tabela I. Parâmetros faunísticos das espécies de moscas frugívoras (Diptera: Tephritoidea) capturadas com armadilhas McPhail nas reservas florestais naturais Sítio Pingo de Amor (mata decídua) e Canal do Tamengo (mata ciliar), município de Corumbá-MS (agosto de 2003 a agosto de 2004).

\begin{tabular}{|c|c|c|c|c|c|c|c|c|c|c|c|c|c|}
\hline \multirow[t]{2}{*}{ Espécies } & \multicolumn{6}{|c|}{ Mata Decídua } & \multicolumn{6}{|c|}{ Mata Ciliar } & \multirow[t]{2}{*}{ Tota } \\
\hline & $\mathrm{N}$ & $\%$ & A & $\mathrm{F}$ & $\mathrm{C}$ & $\mathrm{D}$ & $\mathrm{N}$ & $\%$ & A & $\mathrm{F}$ & $\mathrm{C}$ & $\mathrm{D}$ & \\
\hline \multicolumn{14}{|l|}{ Tephritidae } \\
\hline A. dissimilis Stone 1942 & 1 & 0,70 & $\mathrm{~d}$ & $\mathrm{pf}$ & $\mathrm{z}$ & $\mathrm{n}$ & 0 & 0,00 & - & - & - & - & 1 \\
\hline A.fraterculus (Wied. 1830) & 29 & 20,28 & $\mathrm{a}$ & $\mathrm{mf}$ & $\mathrm{y}$ & $\mathrm{s}$ & 16 & 8,51 & $\mathrm{c}$ & $\mathrm{f}$ & $\mathrm{y}$ & $\mathrm{n}$ & 45 \\
\hline A. obliqua (Macquart 1835) & 59 & 41,26 & $\mathrm{ma}$ & $\mathrm{mf}$ & $\mathrm{y}$ & s & 103 & 54,79 & $\mathrm{ma}$ & $\mathrm{mf}$ & $\mathrm{y}$ & $\mathrm{n}$ & 162 \\
\hline A. rheediae Stone 1942 & 1 & 0,70 & $\mathrm{~d}$ & $\mathrm{pf}$ & $\mathrm{z}$ & $\mathrm{n}$ & 0 & 0,00 & - & - & - & - & 1 \\
\hline A. sororcula Zucchi 1979 & 34 & 23,78 & $\mathrm{a}$ & $\mathrm{mf}$ & $\mathrm{w}$ & $\mathrm{s}$ & 39 & 20,74 & $\mathrm{c}$ & $\mathrm{mf}$ & $\mathrm{y}$ & $\mathrm{n}$ & 73 \\
\hline A. undosa Stone 1942 & 1 & 0,70 & $\mathrm{~d}$ & $\mathrm{pf}$ & $\mathrm{z}$ & $\mathrm{n}$ & 0 & 0,00 & - & - & - & - & 1 \\
\hline C. capitata (Wied. 1824) & 18 & 12,58 & $\mathrm{c}$ & $\mathrm{mf}$ & $\mathrm{y}$ & $\mathrm{n}$ & 30 & 15,96 & $\mathrm{c}$ & $\mathrm{mf}$ & $\mathrm{y}$ & $\mathrm{n}$ & 48 \\
\hline Total & 143 & 100 & & & & & 188 & 100 & & & & & 331 \\
\hline \multicolumn{14}{|l|}{ Lonchaeidae } \\
\hline Dasiops sp.1 & 1 & 1,64 & $\mathrm{~d}$ & $\mathrm{pf}$ & $\mathrm{z}$ & $\mathrm{n}$ & 1 & 4,54 & $\mathrm{~d}$ & $\mathrm{pf}$ & $\mathrm{z}$ & $\mathrm{n}$ & 2 \\
\hline Dasiops sp.2 & 2 & 3,28 & $\mathrm{c}$ & $\mathrm{f}$ & $\mathrm{z}$ & $\mathrm{n}$ & 0 & 0,00 & - & - & - & - & 2 \\
\hline Lonchaea sp.1 & 3 & 4,92 & $\mathrm{c}$ & $\mathrm{f}$ & $\mathrm{z}$ & $\mathrm{n}$ & 4 & 18,20 & $\mathrm{c}$ & $\mathrm{mf}$ & $\mathrm{z}$ & $\mathrm{n}$ & 7 \\
\hline Lonchaea sp.2 & 21 & 34,43 & $\mathrm{c}$ & $\mathrm{mf}$ & $\mathrm{z}$ & $\mathrm{s}$ & 5 & 22,72 & $\mathrm{c}$ & $\mathrm{mf}$ & $\mathrm{z}$ & $\mathrm{n}$ & 26 \\
\hline Neosilba sp.1 & 29 & 47,54 & $\mathrm{ma}$ & $\mathrm{mf}$ & $\mathrm{y}$ & $\mathrm{s}$ & 12 & 54,54 & $\mathrm{a}$ & $\mathrm{mf}$ & $\mathrm{y}$ & $\mathrm{s}$ & 41 \\
\hline Neosilba sp.2 & 5 & 8,19 & $\mathrm{c}$ & $\mathrm{f}$ & $\mathrm{z}$ & $\mathrm{n}$ & 0 & 0,00 & - & - & - & - & 5 \\
\hline Total & 61 & 100 & & & & & 22 & 100 & & & & & 83 \\
\hline
\end{tabular}

$\mathrm{N}$ : Número de indivíduos capturados.

A: abundância - ma = muito abundante, $\mathrm{a}=$ abundante, $\mathrm{c}=$ comum, $\mathrm{d}=$ dispersa.

$\mathrm{F}$ : freqüência $-\mathrm{mf}=$ muito freqüente, $\mathrm{f}=$ freqüente, $\mathrm{pf}=$ pouco freqüente.

$\mathrm{C}$ : Constância $-\mathrm{w}=$ constante, $\mathrm{y}=$ acessória, $\mathrm{z}=$ acidental.

$\mathrm{D}$ : dominância $-\mathrm{s}=$ dominante, $\mathrm{n}=$ não dominante.

(manga), Spondias purpurea L. (serigüela) e Spondias lutea L. (cajá-mirim), nas proximidades das áreas estudadas, sendo estas frutíferas suas hospedeiras preferenciais (Zucchi 2000). Entretanto, Silva \& Ronchi-Teles (2000), observaram na Região Norte do Brasil que A.obliqua coloniza também frutos de algumas espécies de Myrtaceae. Zahler (1990), no Distrito Federal, em estudo de flutuação populacional de moscas-dasfrutas capturadas em armadilhas, também encontrou esta espécie como a mais freqüente.

A. sororcula foi predominante na mata decídua. Em Mato Grosso do Sul esta espécie infesta principalmente frutos de Myrtaceae, tais como: guavira (Campomanesia spp.), araçá, goiaba (Psidium spp.), cagaita (Eugenia dysinterica D.C.), entre outras (Uchôa-Fernandes et al. 2002); todas ocorrentes na região. Como destacado por Canal et al. (1988), a predominância de uma espécie de Anastrepha em determinado local está diretamente associada à presença de seus frutos hospedeiros.

A. fraterculus é considerada a espécie mais polífaga de Anastrepha no Brasil, e também, uma das mais abundantes em pomares do nordeste, sudeste (Nascimento et al. 1983, Martins et al. 2000, Souza-Filho et al. 2000) e sul do País (Kovaleski et al. 1999, Garcia et al. 2003). No entanto, neste estudo foi apenas a terceira em freqüência $(20,28 \%)$ na mata decídua (Tabela I).

De Lonchaeidae, apenas Neosilba sp.1 foi caracterizada como espécie muito abundante na mata decídua, provavelmente, devido à grande abundância de frutos de pequi (Caryocar brasiliense Camb.- Caryocaraceae), um dos seus hospedeiros (Uchôa-Fernandes et al. 2002). Na mata ciliar não ocorreram espécies de lonqueídeos muito abundantes (Tabela
I). No sudoeste de Mato Grosso do Sul espécies de Neosilba foram capturadas ininterruptamente durante 25 meses de amostragens (Uchôa-Fernandes et al. 2003b). Algumas espécies deste gênero colonizaram 22 espécies de frutos silvestres ou cultivados, com predominância em Citrus spp. e pequi (Uchôa-Fernandes et al. 2002).

O menor índice de diversidade encontrado na mata ciliar pode ser explicado pelo fato daquela área ser comparativamente mais afetada pelas inundações periódicas via Rio Paraguai. Isto pode prejudicar o desenvolvimento da fase juvenil, visto que essas moscas utilizam o solo para seu desenvolvimento pupal. De acordo com Ronchi-Teles \& Silva (2005), a diminuição na aeração do solo em períodos de elevada precipitação pode constituir um importante fator de mortalidade para as pupas. Silveira Neto et al. (1976), ponderam que os valores do índice de diversidade tendem a ser baixos em locais onde fatores limitantes e competição interespecífica atuam intensamente.

As baixas diversidade e abundância das moscas frugívoras no Pantanal são, provavelmente, devidas ao regime de inundações periódicas. Isso pode ocasionar a mortalidade das larvas que se enterram no solo para empupar, pois, em várias ocasiões coincidentes com períodos de pós-inundação, foram instaladas armadilhas McPhail iscadas com atrativo alimentar, sem sucesso na captura de adultos (Uchôa-Fernandes, M. A., dados não publicados). No entanto, há necessidade de estudos com metodologia específica, visando a amostragem das populações de Tephritoidea em períodos de seca, de enchentes e em áreas de planalto nas proximidades do Pantanal, para esclarecer este aspecto sobre as espécies de 
moscas frugívoras nas regiões periodicamente alagadas.

Agradecimentos. À coordenação de Aperfeiçoamento de Pessoal de Nível Superior (CAPES) pela concessão de bolsa de estudo ao primeiro autor; à Fundação de Apoio ao Desenvolvimento do Ensino, Ciência e Tecnologia do Estado de Mato Grosso do Sul (FUNDECT) pelo aporte financeiro ao projeto "Biodiversidade de Insetos Frugívoros, seus Hospedeiros e Inimigos Naturais no Brasil Central"; $15^{\circ}$ Batalhão de Polícia Militar Ambiental de Mato Grosso do Sul (XV BPMA-MS) e soldado Arlei de Souza Machado, pelo auxílio às coletas no campo e ao Prof. Dr. Marcos Gino Fernandes-FCBA-UFGD, pelo auxílio nas análises dos parâmetros faunísticos.

\section{REFERÊNCIAS}

Aluja, M.; J. Rull; J. Sivinski; A. L. Norrbom; R. A. Wharton; R. MacíasOrdóñez; F. Díaz-Fleischer \& M. López. 2003. Frut flies of the genus Anastrepha (Diptera: Tephritidae) and associated native parasitoids (Hymenoptera) in the tropical rainforest biosphere reserve of Montes Azules, Chiapas, Mexico. Environmental Entomology 32: 1377-1385.

Araújo, E. L.; M. K. M. Medeiros; V. E. Silva \& R. A. Zucchi. 2005. Moscas-das-frutas (Diptera: Tephritidae) no semi-árido do Rio Grande do Norte: Plantas hospedeiras e índices de infestação. Neotropical Entomology 34: 889-894.

Bomfim, D. A.; M. A. Uchôa-Fernandes \& M. A. L. Bragança. 2007. Biodiversidade de moscas-das-frutas (Diptera, Tephritoidea) em matas nativas e pomares domésticos de dois municípios do Estado do Tocantins, Brasil. Revista Brasileira de Entomologia 51: 217-223.

Canal, N. A.; C. D. Alvarenga \& R. A. Zucchi. 1988. Análise faunística de moscas-das-frutas (Diptera: Tephritidae) em Minas Gerais. Scientia Agricola 55: $15-25$.

Canesin, A. \& M. A. Uchôa-Fernandes. 2007. Análise faunística e flutuação populacional de moscas-das-frutas (Diptera, Tephritidae) em um fragmento de floresta semidecídua em Dourados, Mato Grosso do Sul, Brasil. Revista Brasileira de Zoologia 24: 185-190.

Colwell, R. K. 2001. Statistical estimation of richness and shared species from samples. Version $6.0 \mathrm{~b} 1$. User's guide and aplication published: http://viceroy.eeb.uconn.edu/estimates. Acesso em dezembro de 2005.

Duarte, A. L. \& A. Malavasi. 2000. Tratamentos quarentenários, p. 187-192. In: Malavasi, A. \& R. A. Zucchi (eds.). Moscas-dasfrutas de importância econômica no Brasil: conhecimentos básico e aplicado. Ribeirão Preto, Holos Editora, 327 p.

Duyck, P. F.; P. David \& S. Quilici. 2004. A review of relationships between interspecific competition and invasions in fruit flies (Diptera: Tephritidae). Ecological Entomology 29: 511-520.

Garcia, F. R. M.; J. V. Campos \& E. Corseuil. 2003. Análise faunística de espécies de moscas-das-frutas (Diptera: Tephritidae) na Região Oeste de Santa Catarina. Neotropical Entomology 32: 421-426.

Hernández-Ortiz, V. \& R. Pérez-Alonso, 1993. The natural host plants of Anastrepha (Diptera: Tephritidae) in a tropical rainforest of Mexico. Florida Entomologist 76: 447-460.

Kovaleski, A.; R. L. Sugayama; N. A. Canal-Daza \& A. Malavasi. 1999. A survey of Anastrepha Schiner (Diptera, Tephritidae) species in the apple growing area of the state of Rio Grande do Sul, Brazil. Revista Brasileira de Entomologia 43: 229-234.

Martins, D. dos. S.; K. Uramoto \& A. Malavasi. 2000. Moscas-dasfrutas nos estados brasileiros: Espírito Santo, pp. 253-258. In: Malavasi, A. \& R. A. Zucchi (eds.). Moscas-das-frutas de importância econômica no Brasil: conhecimentos básico e aplicado. Ribeirão Preto, Holos Editora, 327 p.

McAlpine, J. F. 1993. Manual of Nearctic Diptera. Vol. 2. Canada Agriculture. Canada Communication Group Publishing, Ottawa. $1.132 \mathrm{p}$.

Nascimento, A. S.; R. A. Zucchi \& S. Silveira Neto, 1983. Dinâmica populacional das moscas-das-frutas no Recôncavo Baiano. III. Analise faunística. Pesquisa Agropecuária Brasileira 18: 319328.

Niklaus-Ruiz, M. \& T. Basedow. 1997. A survey on the occurrence and flight periods of fruit fly species (Diptera: Tephritidae) in a fruit growing area in southwest Nicaragua, 1994/95. Bulletin of Entomological Research 87: 405-412.

Ovruski, S. M.; R. A. Wharton; P. Schliserman \& M. Aluja. 2005. Abundance of Anastrepha fraterculus (Diptera: Tephritidae) and its associated native parasitoids (Hymenoptera) in "Feral" guavas groving in the endangered Northernmost yungas forests of Argentina with an update on the taxonomic status of Opiine parasitoids previously reported in this country. Environmental Entomology 34: $807-818$

Pott, A. \& J. V. Pott. 1994. Plantas do Pantanal. Brasília, EMBRAPASPI, $320 \mathrm{p}$.

Price, P. W. 1997. Insect Ecology. pp. 659-662. $3^{\text {rd }}$. Edition. New York. John Wiley \& Sons. 874 p.

Ravazzani C.; H. Wiederkehr-Filho; J. P. Fagnani \& S. da Costa. 1991. Pantanal Brazilian Wildlife. Curitiba. Editora Edibran. 180 p.

Ronchi-Teles, B. \& N. M. da Silva. 2005. Flutuação populacional de espécies de Anastrepha Schiner (Diptera: Tephritidae) na região de Manaus, AM. Neotropical Entomology 34: 733-741.

Silva, N. M. \& B. Ronchi-Teles. 2000. Moscas-das-frutas nos estados brasileiros: Amapá, Amazonas, Pará, Rondônia e Roraima, pp.203209. In: Malavasi, A. \& R. A. Zucchi. (eds.). Moscas-das-frutas de importância econômica no Brasil: conhecimentos básico e aplicado. Ribeirão Preto, Holos Editora, 327 p.

Silveira Neto, S.; O. Nakano; D. Barbin \& N. A. Villa Nova. 1976. Manual de ecologia dos insetos. São Paulo, Editora Agronômica Ceres, $420 \mathrm{p}$.

Souza-Filho, M. F.; A. Raga \& R. A. Zucchi. 2000. Moscas-das-frutas nos estados brasileiros: São Paulo. pp. 277-283. In: Malavasi, A. \& R. A. Zucchi (eds.). Moscas-das-frutas de importância econômica no Brasil: conhecimentos básico e aplicado. Ribeirão Preto, Holos Editora, 327 p.

Uchôa-Fernandes, M. A; I. de Oliveira; R. M. S. Molina \& R. A. Zucchi. 2002. Species diversity of frugivorous flies (Diptera: Tephritoidea) from hosts in the cerrado of the State of Mato Grosso do Sul, Brazil. Neotropical Entomology 31: 515-524.

Uchôa-Fernandes, M. A; I. de Oliveira; R. M. S. Molina \& R. A. Zucchi. 2003a. Biodiversity of frugivorous flies (Diptera: Tephritoidea) captured in citrus groves, Mato Grosso do Sul, Brazil. Neotropical Entomology 32: 239-246.

Uchôa-Fernandes, M. A; I. de Oliveira; R. M. S. Molina \& R. A. Zucchi. 2003b. Populational fluctuation of frugivorous flies (Diptera: Tephritoidea) in two orange groves in the State of Mato Grosso do Sul, Brazil. Neotropical Entomology 32: 19-25.

Zahler, P. M. 1990. Moscas-das-frutas em três pomares do Distrito Federal: levantamento de espécies e flutuação populacional. Ciência e Cultura 42: 177-182.

Zucchi, R. A. 2000. Taxonomia, pp. 13-24. In: Malavasi, A. \& R. A. Zucchi (eds.). Moscas-das-frutas de importância econômica no Brasil: conhecimentos básico e aplicado. Ribeirão Preto, Holos Editora, 327 p. 